\title{
FMRI activity patterns in human LOC carry information about object exemplars within category
}

\author{
Evelyn Eger ${ }^{1,2}$, John Ashburner ${ }^{2}$, John-Dylan Haynes ${ }^{1,2}$, Raymond J Dolan ${ }^{2}$, and Geraint \\ Rees $^{1,2}$ \\ ${ }^{1}$ Institute of Cognitive Neuroscience, University College London, 17 Queen Square, London \\ WC1N 3AR \\ ${ }^{2}$ Wellcome Department of Imaging Neuroscience, Institute of Neurology, University College \\ London, 12 Queen Square, London WC1N 3BG
}

\begin{abstract}
The lateral occipital complex (LOC) is a set of areas in human occipito-temporal cortex responding to objects as opposed to low-level control stimuli. Conventional fMRI analysis methods based on regional averages could not detect signals discriminative of different types of objects in this region. Here, we examined fMRI signals using multivariate pattern recognition (support vector classification), to systematically explore the nature of object-related information available in fine-grained activity patterns in LOC. Distributed fMRI BOLD signals from LOC allowed for above chance discrimination not only of the category, but also of within-category exemplars of everyday man-made objects, and such exemplar-specific information generalised across changes in stimulus size and viewpoint, particularly in posterior subregions. Object identity could also be predicted from responses of the early visual cortex, even significantly across the changes in size and viewpoint used here. However, a dissociation was observed between these two regions of interest in the degree of discrimination for objects relative to size: In the early visual cortex two different sizes of the same object were even better discriminated than two different objects (in accordance with measures of pixelwise stimulus similarity), whereas the opposite was true in the LOC. These findings provide the first evidence that direct evoked fMRI activity patterns in LOC can be different for individual object exemplars (within a single category). We propose that pattern recognition methods as used here may provide an alternative approach to study mechanisms of neuronal representation based on aspects of the fMRI response independent of those assessed in adaptation paradigms.
\end{abstract}

\section{Keywords}

object representation; fMRI; LOC; invariance; SVM

\footnotetext{
Correspondence and requests for materials to: Dr. Evelyn Eger INSERM U.562, SHFJ-CEA 4 place du General Leclerc Orsay Cedex, France Telephone: +33 (0) 169867840 Fax: +33 (0) 169867816 evelyn.eger@ gmail.com.

${ }^{1}$ Because ROIs were based on spheres around the activation maxima, one potential factor contributing to inferior classification performance in anterior subregions could be that this region is situated close to areas of MR susceptibility artifact, and this, classification could have included voxels where signal is very low. To rule out this possibility, analysis was repeated using only voxels within the chosen spheres that showed a significant difference between object and scrambled pictures in the localizer scan, which yielded qualitatively unchanged results. Likewise, restriction of the analysis to gray matter voxels using a gray matter mask derived from SPM segmentation did not change results, indicating that any inclusion of white matter voxels in our ROIs did not affect the findings.
} 


\section{Introduction}

Two photographs of the same object taken from different viewpoints, or under different lighting conditions, may show little similarity in their low-level physical properties. Despite this, our visual system can extract a representation of the object abstract enough to overcome such low-level differences, but specific enough to discriminate from a similar object of the same category. The computational mechanisms underlying this ability and their implementation in the human brain are still poorly understood. In humans, a complex of areas in the lateral occipital and inferior temporal cortex, termed the 'lateral occipital complex' (LOC), is more active when viewing objects compared to textures or scrambled images (Malach et al., 1995; Grill-Spector, 2003). The LOC is considered a structure subserving general shape processing (common to all object types), but the precise nature of object-selective information within the LOC (i.e. in how far, and at what level of representation, LOC distinguishes individual objects) remains to be established. Population measures of neural activity such as functional magnetic resonance imaging (fMRI) are often considered unhelpful in resolving this type of question given the evidence that at the level of single neurons objects are likely to be represented in a distributed and spatially overlapping fashion (Rolls \& Tovee, 1995; Logothetis, Pauls, \& Poggio, 1995; Young \& Yamane, 1992; Gross, 1992; Hung, Kreiman, Poggio, \& DiCarlo, 2005) that is beyond the spatial resolution of conventional fMRI.

Indirect methods, such as repetition priming or fMR adaptation paradigms, (e.g., GrillSpector et al., 1999; Kourtzi \& Kanwisher, 2000; 2001; Avidan, Hasson, Hendler, Zohary, \& Malach, 2002; James, Humphrey, Gati, Menon, \& Goodale, 2002; Vuilleumier, Henson, Driver, \& Dolan, 2002; Sawamura, Georgieva, Vogels, Vanduffel, \& Orban, 2005), have therefore been used to probe the nature of object representations in LOC, but often with inconsistent findings. For example, studies differ in whether they suggest size and viewpoint invariant representation of objects in different subparts of LOC (Grill-Spector et al., 1999; James, Humphrey, Gati, Menon, \& Goodale, 2002; Vuilleumier, Henson, Driver, \& Dolan, 2002; Sawamura, Georgieva, Vogels, Vanduffel, \& Orban, 2005). Common to these studies is the reliance on a repetition-associated change in activation (usually blood oxygenation level-dependent BOLD signal decrease) between consecutive presentations of the same (or a related) stimulus. Although these effects on the BOLD signal have been assumed to either reflect neural adaptation or some other change in the neural representation of the stimulus, the exact mechanisms are as yet unknown and probably complex (Grill-Spector, Henson, \& Martin, 2006; Krekelberg, Boynton, \& Van Wezel, 2006). Because reliance on repetitionassociated activity changes implies reliance on some form of mnemonic phenomenon, different mechanisms might be involved depending on the temporal lags between repetitions (Henson, 2003). These in turn may interact to a greater or lesser degree with task requirements (Henson, Shallice, Gorno-Tempini, \& Dolan, 2002) or other factors, such as level of attention during repetition (Murray \& Wojciulik, 2004; Eger, Henson, Driver, \& Dolan, 2004; Vuilleumier, Schwartz, Duhoux, Dolan, \& Driver, 2005, Yi \& Chun, 2005).

In the light of these limitations in existing paradigms, here we sought to address the nature of LOC responses using an alternative approach, based on direct evoked fMRI activity (instead of changes of such activity due to repetition). Multivariate pattern classification has previously been used to show that object category information can be reflected in distributed activity patterns across extended areas of inferotemporal cortex (Haxby et al., 2001; Carlson, Schrater, \& He, 2003; Cox \& Savoy, 2003, O'Toole, Jiang, Abdi, \& Haxby, 2005). These studies were restricted to the discrimination of different object categories, not testing for any potential difference of response to exemplars within category, and thus leaving open the extent to which effects reflect the representation of visual features per se (instead of, e.g., semantic differences associated with categories). More recently, pattern recognition 
techniques have been applied successfully to individual visual areas in earlier visual cortex. For example, information sufficient to reconstruct the orientation of a stimulus can be decoded from human V1 (Kamitani \& Tong, 2005; Haynes \& Rees, 2005) even though the spatial layout of orientation-columns is below the spatial resolution of conventional fMRI.

We therefore hypothesised that such techniques might also provide more detailed insights into the nature of object representation within more circumscribed higher-level visual areas such as LOC, even beyond discriminating responses to images of different categories. Conventional methods using average signals could not detect selective responses to different types of objects in this region, leading to the notion that this area may not be discriminative of different objects, and only involved in object detection (Malach et al., 1995; GrillSpector, 2003). In a series of three experiments, we used multivariate pattern recognition to systematically explore whether distributed signals from this area carried information sufficient to discriminate object category, as well as the identity of individual exemplars of two everyday man-made categories presented in different sizes or from different viewpoints. Furthermore, we compared discrimination results obtained in LOC for different objects, sizes and views, as well as generalisation across changes in size and view, with those obtained in early visual cortex (area 17/V1). Finally, we related our new fMRI findings to classification results and measures of low-level similarity based on the stimuli themselves.

\section{Methods}

\section{Participants and Data acquisition}

Eighteen healthy right-handed volunteers with normal or corrected vision (7 male and 11 female, mean age $27.3+/-5.4$ years) gave written informed consent to participate in the three separate experiments, which were approved by the local ethics committee. Functional images were acquired on a 3 Tesla MR system with standard head coil (Siemens Allegra, Erlangen, Germany) as T2* weighted echo-planar image (EPI) volumes with a TR of $1.4 \mathrm{~s}$ (TE $30 \mathrm{~ms}, 22$ transverse slices, voxel size $3 \times 3 \times 2 \mathrm{~mm}$, skip $1 \mathrm{~mm}$ ).

\section{Stimuli and Design}

Stimuli (Figure 1) were created from 3D models that were either freely available at various internet sites, or created from scratch in Blender 2.3 (http://www.blender.org). All object models ( 4 chairs and 4 teapots) were equipped with the same surface texture of a uniform gray colour, and illuminated with the same single light source. For the viewpoint experiment, two views were created separated by $60 \mathrm{deg}$ rotation around the vertical axis, while avoiding strongly un-canonical views and occlusion of parts. In Experiment 1 and 3, rendered views subtended $200 \times 200$ pixels, while in Experiment 2 the small stimuli subtended $160 \times 160$ pixels, and the large stimuli subtended $240 \times 240$ pixels.

In Experiment 1, all participants were presented with pictures of chairs and teapots. In Experiments 2 and 3, half the participants were presented with the four teapots, and the other half the four chairs as stimuli (in two sizes, or two viewpoints each) to restrict the overall number of experimental conditions to eight.

\section{Experimental protocol and task}

Stimuli were back-projected onto a translucent screen located $\sim 60 \mathrm{~cm}$ above the subjects' head and viewed via a mirror on the head coil. The presented pictures subtended $\sim 5^{\circ}$ of visual angle. Objects were presented in short blocks of 4 pictures each ( 1 per TR, $1 \mathrm{~s}$ stimulus, $0.4 \mathrm{~s}$ blank), followed by a fixation baseline of 3 TR (Figure 1). The order of conditions was pseudo-randomised. Within a given short block each stimulus could randomly appear in a red or green hue, and participants were required to press one of two 
buttons on a keypad, depending on the colour of the current stimulus. Five experimental sessions of 9.5 mins length each were run for each participant with this task, encompassing 35 short blocks of each experimental condition.

In an additional scanning session of $\sim 6$ mins length, object-responsive areas were determined for all participants using a standard LOC localiser (Grill-Spector, 2003), comparing pictures of various common objects to scrambled versions of the same pictures in a blocked presentation with 500 ms per picture every $1 \mathrm{~s}$, and block length of $12 \mathrm{~s}(6 \mathrm{~s}$ fixation baseline) during which participants performed a one-back repetition detection task. This functional localiser was used for definition of regions of interest (see Figure 2) for the specific classification-based fMRI analysis performed, as described below.

\section{Image processing and data analysis}

Initial analysis of the imaging data was performed in SPM2 (http://www.-fil.ion.ucl.ac.uk/ spm2.html). After motion correction, the un-normalised and un-smoothed EPI-images were entered into a general linear model, modelling separately the effect of each short block (35/ condition) convolved with a standard hemodynamic response function, while accounting for serial autocorrelation with an $\mathrm{AR}(1)$ model and removing low frequency drift terms by a high-pass filter with cutoff $128 \mathrm{~s}$. This analysis yielded 35 independent estimates of BOLD signal change (images of regression coefficients) for each condition, which were subsequently entered into pattern recognition analysis.

We used a two stage procedure to define regions of interest based on a standard independent 'LOC localiser' scan (e.g., Grill-Spector, 2003). First, we identified voxels in ventral occipito-temporal cortex in each participant that showed significantly greater responses to visually presented objects versus scrambled objects (Figure 2). In line with previous findings, posterior and anterior subregions in lateral occipital and fusiform cortex were apparent in this contrast (see Figure 2 for mean stereotactic coordinates). We then identified the response maxima in these anterior and posterior regions on a per-participant basis and defined spherical regions of interest of $10 \mathrm{~mm}$ radius around these maxima. A spherical restriction of ROIs was used as a means to obtain comparable numbers of voxels across participant (as desirable for multivariate analysis) without the need for participant-specific thresholding. We further confirmed that regions of interest in anterior and posterior LOC were non-overlapping, and that any regions of white matter included in the regions of interest did not affect our findings. For the left and right ROIs together, these comprised on average $212(+/-37)$ voxels for the posterior, and $275(+/-29)$ voxels for the anterior part across participants (the exact number of voxels differing slightly because not all voxels within the spheres contained data in each case, e.g. due to signal drop-out or location outside of the brain mask). For the initial analyses anterior and posterior ROIs were combined while for subsequent analyses we compared anterior and posterior sampled sub-regions of LOC.

For the analysis of early visual cortex, regions of interest were defined by a mask based on a probabilistic map of area 17/V1 in MNI-space, derived from the Anatomy toolbox for SPM (http://www.fz-juelich.de/ime/spm_anatomy_toolbox). This mask was back-transformed onto the individual subjects brains (Deformation toolbox for SPM) to create individual ROIs for each participant.

Pattern recognition analysis was used to predict from distributed response patterns in these ROIs which of two given stimuli was currently being presented. The BOLD signal change estimates of $\mathrm{n}$ voxels were extracted for each condition and for repeated blocks forming a set of pattern vectors $\mathbf{x}$ which can be considered as points in an n-dimensional Euclidean space. Pattern classification was performed using linear support vector machines (SVM - Vapnik, 
1995; Christianini \& Shawe-Taylor, 2000) in the implementation of Gunn (http:// www.isis.ecs.soton.ac.uk/). A linear classifier finds a hyperplane

$$
\mathbf{w}^{\mathrm{T}} \mathbf{x}+\mathrm{b}=0
$$

defined by weight vector $\mathbf{w}$ and offset $\mathrm{b}$ separating the training points $\mathbf{x}$ with two different given labels. The principle of SVM is to find the optimally separating hyperplane that maximises the margin (given by $2 /\|\mathbf{w}\|$ ) with respect to both training classes (see Christianini \& Shawe-Taylor, 2000, for detailed algorithm). Under the presence of noise (as here) the response vectors of both stimuli might not be linearly separable and a so-called "soft-margin classifier" can be used which allows for a certain proportion of misclassifications by minimising

$$
\|\mathbf{w}\| / 2+\mathrm{C} 3 \xi_{\mathrm{i}}
$$

subject to

$$
\mathrm{y}_{\mathrm{i}}\left(\mathbf{w} \mathbf{x}_{\mathrm{i}}+\mathrm{b}\right) \exists 1-\xi_{\mathrm{i}} \quad \mathrm{i}=1,2, \ldots, \mathrm{N} \quad \xi \exists 0
$$

where $\xi_{\mathrm{i}}$ is a slack variable representing misclassification error for the $\dot{t}$ th pattern $\mathbf{x}_{\mathrm{i}}$ with label $\mathrm{y}_{\mathrm{i}} 0\{1,-1\}$ and $\mathrm{C}$ a regularisation parameter determining the tradeoff between largest margin and lowest number of misclassifications. In the analysis presented here, a linear softmargin classifier with $\mathrm{C}=5$ was used throughout (value of $\mathrm{C}$ chosen based on literature and not tested for optimality with present data). The classifier was trained on all but one $(=34)$ replications for two given experimental conditions, and subsequently tested on the remaining data, with 35 possible assignments of independent training and test data sets (leave-one-out prediction with 35 -fold cross-validation).

The number of training and test data in each pairwise comparison was identical in object, size, and view comparisons. Classification accuracies were computed across all different training and test data assignments as mean percent correct for the two objects in a given pair. This mean pairwise percent correct is independent of classifier bias, comparable to the signal detection measure $\mathrm{d}^{\prime}$ in a choice reaction task as performed here by the classifier. Classification accuracies were subsequently averaged across all possible pairwise comparisons to yield mean classification performance (for e.g. objects across categories, within category, size, or view). The theoretical chance level in this case corresponds to 50 $\%$, which was further confirmed by performing classification on data with randomised labels, see Figures 3, 4, 5, and 7. Significance of classification performance was assessed in two-tailed t-tests (against chance $=50 \%$ ) across the 6 subjects in each experiment. This, in addition to identical training and test conditions (see above), should ensure the results are comparable across the different comparisons between objects, sizes and views. While comparisons across different brain regions are informative in showing relative differences between different types of comparisons (e.g. size discrimination and object discrimination), absolute effect sizes across regions may be less comparable, since they are sensitive to different signal-to-noise ratios and other factors unrelated to the biological question under study.

For the analyses investigating prediction accuracies as a function of number of voxels included, voxels were rank-ordered based on their absolute pairwise t-value for a given comparison of interest, derived from the training data only (identical for the conditions testing for discrimination of the same image, and generalisation). This analysis was 
conducted for $1,5,10,20,40$, and further in steps of 40 up to 400 voxels. Due to the pairwise implementation of this selection, different voxels might be selected first in different pairwise comparisons between condition.

\section{Results}

In the first experiment, we investigated whether signals from LOC carried information sufficient to determine either object category or the identity of individual exemplars from that category. We presented participants with four successive pictures of the same view of a particular object, in short blocks interleaved with short baseline periods (Figure 1A), while brain activity was measured with functional MRI (see Methods). Four different exemplars of everyday man-made objects from each of two different categories (teapots and chairs, one view for each object, see Figure 1B) were used, with different objects presented in different blocks. Participants' attention was engaged in a colour judgement task for each object, which could randomly appear in a red or green hue.

BOLD contrast responses evoked by each object in the main experiment in the sampled subregions of LOC were extracted separately for each block (see Methods). To determine whether distributed response patterns in the chosen ROIs could successfully discriminate between and within different object categories, we then applied a multivariate pattern recognition algorithm based on Support Vector Machines (see Methods). To compare the performance of our multivariate technique with more conventional analyses, we also determined whether successful prediction could be achieved based on the mean level of activation in the sampled LOC sub-regions, or on just a few maximally stimulus-selective voxels. Prediction accuracies tended to increase with larger numbers of voxels and plateau at around 200 voxels. Below we focus on reporting results for 1, 5, 10, 200, and all 400 voxels, while full results for all intermediate steps can be found in Supplemental Materials (Supplemental Figure S1).

Classification accuracy was significantly above chance for both between and within category comparisons when using 200 (between: $\mathrm{t}(5)=4.84, \mathrm{p}<.01$, within: $\mathrm{t}(5)=9.65, \mathrm{p}<$. 001 ) or the maximum of 400 (between: $t(5)=4.31, p<.01$, within: $t(5)=6.19, p<.01)$ voxels). In contrast, pairwise classification accuracy was at chance when based on mean activity for both comparisons. Accuracy was also not significantly different from chance (across subjects) for within category comparisons when using the 1 to 10 most discriminative voxels, indicating that the fMRI pattern signal upon which successful classification was based encompassed relatively large areas of LOC. Thus, although the mean signal in LOC (as for conventional fMRI analyses) did not discriminate between and within object categories, the multivariate pattern signal now allowed successful classification performance. This indicates that neural population responses within human $\mathrm{LOC}^{1}$, in addition to discriminating between categories, also carry information sufficient to discriminate finer detail between individual object exemplars. Finally, although both comparisons were significant, between category prediction accuracies were better (average $62 \%$ at 200 voxels) than within category prediction accuracies (average $55 \%$ at 200 voxels), $\mathrm{t}(5)=3.14, \mathrm{p}<.05$, and between category comparisons were already above chance for up to ten most discriminative voxels (see Figure 3).

\footnotetext{
${ }^{1}$ In addition to regions defined by the LOC localiser, we performed an analysis based on lateral occipital and fusiform clusters activated by objects vs baseline in the main experiments, with similar results (Supplemental Figure 2)
} 


\section{Within-category discrimination and effects of size}

In the second experiment, we determined whether the signals used for successful discrimination within a single category were invariant to stimulus size. A further six participants were now presented with four exemplars from just one of the two categories. However, the individual exemplars were presented in two different sizes $(50 \%$ increase from small to large, see Figure 1C). This experiment thus focused on within-category comparisons alone. Pattern classification proceeded in the same way as in Experiment 1, but we now in addition 1) changed object size between training and test to determine whether information from LOC signals used by our classifier was sufficient to generalise across size, and 2) attempted to directly discriminate the two sizes of each object, to test whether LOC contains information about size in addition to object shape.

When training and test were carried out with data corresponding to the same object in a different size, significant above chance accuracies were obtained for object classification based on higher voxel numbers within LOC (200 voxels, $t(5)=3.50, p<.05,400$ voxels $\mathrm{t}(5)=3.79, \mathrm{p}<.05)$. Thus, pattern signals within human LOC represent information sufficient to identify individual objects across changes in their size. For training and test using the same size objects, we replicated the within-category findings from Experiment 1. Significantly above chance classification performance for within-category discrimination was possible when using either $200, \mathrm{t}(5)=3.53, \mathrm{p}<.05$ or $400, \mathrm{t}(5)=2.51, \mathrm{p}=.05$, voxels, testing with data corresponding to the same size. As before, classification based on the mean signal or the most discriminate voxels was not significantly different from chance (Figure 4). Prediction of object size resulted in on average lower discrimination performance than prediction of object exemplar (see Figure 4), which failed to reach significance for 200 (t(5) $=1.4, \mathrm{p}=.22)$ and $400(\mathrm{t}(5)=2.0, \mathrm{p}=.10)$ voxels.

\section{Within-category discrimination and effects of view}

Having established generalisation of distributed LOC response patterns across changes in stimulus size, in a third experiment we investigated whether signals sampled from LOC showed generalisation across views within a single object category. A further six participants were presented with four exemplars of just one of the two categories. However, each exemplar could be presented from two viewing angles that differed by a sixty degree rotation around the vertical axis (Figure 1D). Analogous to the size experiment, in the different view condition the classifier was trained on one view, and then subsequently tested on the different view of the object, in addition to directly comparing the two views of each object.

Significant above-chance discrimination performance was once again obtained when based on the higher voxel numbers (same view: 200 voxels, $t(5)=4.63, \mathrm{p}<.01,400$ voxels $\mathrm{t}(5)=5.55, \mathrm{p}<.01$, different view: 200 voxels, $\mathrm{t}(5)=3.37, \mathrm{p}<.05,400$ voxels, $\mathrm{t}(5)=3.84, \mathrm{p}<.05)$, see Figure 5. In this experiment prediction based on mean activity derived from the five most predictive voxels reached significance for same views. However, since combining the data for the same image condition across all three experiments did not yield a significant effect (e.g. prediction for identical images based on mean activity across all three experiments $\mathrm{t}(17)=.07, \mathrm{p}=.94)$, we do not place much emphasis on these tendencies observed in Experiment 3 alone. As for object size in Experiment 2, prediction of object view resulted in on average lower discrimination performance than prediction of the object exemplar (Figure 5). For samples of 200 voxels, view discrimination showed a trend for significance $(\mathrm{t}(5)=2.2, \mathrm{p}=.08)$ and was nonsignificant $(\mathrm{t}(5)=1.9, \mathrm{p}=.12)$ for 400 voxels. 


\section{Comparison of posterior and anterior sub-regions of LOC}

The results reported above show that BOLD pattern signals sampled from the LOC contained distributed information sufficient to discriminate between objects of different categories as well as of the same category, while average discrimination performance for sizes and views of the same object was lower. Furthermore, for individual objects, object discrimination generalised across moderate changes in stimulus size and viewpoint. As previous reports have suggested a functional distinction between anterior and posterior subparts of the LOC complex (e.g., Grill-Spector et al., 1999; Sawamura, Georgieva, Vogels, Vanduffel, \& Orban, 2005), we next performed separate analyses for the sampled posterior and anterior portions of LOC (Figure 6). These analyses included all voxels in the posterior (located in lateral/inferior occipital cortex; see above and Methods for full details of sampling) or anterior (located in ventral temporal cortex) ROIs without further preselection.

In Experiment 1 an ANOVA with the factors region (posterior/anterior) and object condition (between category/within category) revealed significant main effects of region, $\mathrm{F}(1,5)=6.72$, $\mathrm{p}<.05$, and object condition, $\mathrm{F}(1,5)=17.4, \mathrm{p}<.01$, without a significant interaction, $\mathrm{F}(1,5)=0.96, \mathrm{p}=.37$. As Figure $6 \mathrm{~A}$ indicates, these results reflected higher classification accuracies for posterior LOC (compared to anterior), and also better accuracies for betweencategory comparisons. A similar ANOVA on the data from Experiment 2 revealed a trend for an effect of region, $F(1,5)=4.7, p=.08$, but no effect of object condition (same size/ different size), $\mathrm{F}(1,5)=0.17, \mathrm{p}=.69$, and no interaction, $\mathrm{F}(1,5)=0.71, \mathrm{p}=0.44$. Again, the observed tendency for significance reflected better discrimination performance in posterior LOC (Figure 6B). Finally, an ANOVA for Experiment 3 showed a significant main effect of region, $F(1,5)=20.6, p<.01$, without a significant effect of object condition (same view/ different view), $\mathrm{F}(1,5)=1.65, \mathrm{p}=.25$, or interaction, $\mathrm{F}(1,5)=3.56, \mathrm{p}=.12$, once again indicating better classification performance for posterior than anterior LOC (Figure 6C).

Separate ANOVAs were conducted to assess effects of discrimination type (object versus size/view) across regions. In Experiment 2, this ANOVA revealed a significant main effect of discrimination type, $\mathrm{F}(1,5)=59.0, \mathrm{p}<.001$, indicating better discrimination for different objects in the same size than for different sizes, without neither a significant effect of region, $F(1,5)=13.7, p=.16$, nor interaction, $F(1,5)=7.4, p=.34$. In Experiment 3, there was a trend for a main effect of region, $F(1,5)=5.0, p=.08$, while the main effect of discrimination type, $\mathrm{F}(1,5)=.49, \mathrm{p}=.50$, and the interaction, $\mathrm{F}(1,5)=1.4, \mathrm{p}=.30$, remained non-significant.

Taken together, the results of our comparisons between sampling anterior and posterior LOC reveal consistently better prediction accuracies for sampled data from the posterior LOC. Although collapsed across all three experiments, prediction accuracies for identical images within categories reached significance for the anterior $\operatorname{LOC}(\mathrm{t}(17)=3.14, \mathrm{p}<.01)$, mean classification accuracy was only marginally above chance $(52 \%)^{2}$. This analysis further confirmed higher discrimination performance for different objects than different sizes of the same object, while this did not reach significance for the comparison object versus view.

\footnotetext{
${ }^{2}$ Since ROIs were based on spheres around the activation maxima, one potential factor contributing to inferior classification performance in anterior subregions could be that this region is situated close to areas of MR susceptibility artefact, and thus classification could have included voxels where signal is very low. To rule out this possibility, analysis was repeated using only voxels within the chosen spheres that showed a significant difference between object and scrambled pictures in the localiser scan. Results were qualitatively unchanged (see Supplemental Table 1 for details of accuracy values in the 3 experiments). Likewise, restriction of the analysis to grey-matter voxels using a grey-matter mask derived from SPM segmentation did not change results, indicating that any inclusion of white matter voxels in our regions of interest did not affect the findings (see Supplemental Table 2 for accuracy values).
} 


\section{Occipito-temporal Regions of Interest Defined in Main Experiments}

The use of independent functional localizer sessions (as here for the LOC) has recently been debated controversially (Friston, Rotshtein, Geng, Sterzer, \& Henson, 2006; Saxe, Brett \& Kanwisher, 2006). For this reason, we also conducted an alternative analysis where ROIs were defined on the basis of lateral occipital and fusiform clusters activated in the main experiment (for Experiment 1). Because objects were presented here against a baseline of fixation, this contrast is likely to include voxels that respond to any visual stimulus and not necessarily more strongly to objects as opposed to scrambled objects. For pattern recognition analysis, individual clusters activated for objects > baseline were indentified in lateral occipital and fusiform regions, after which data were sampled and analyzed from these regions in a way analogous to the analysis based on the LOC localizer. The regions of interest defined on the basis of the main experiment were on average located somewhat more posterior than those defined by the LOC localizer, in particular for the anterior subregion (mean difference in $y$ coordinates $4 \mathrm{~mm}$ for the posterior, and $10 \mathrm{~mm}$ for the anterior ROIs).

Analysis of occipito-temporal ROIs defined in this way yielded similar results with, on average, only slightly higher prediction accuracies ( $\sim 64 \%$ correct for discrimination between categories, $\sim 58 \%$ correct for discrimination within categories when selecting from all voxels, and for the same comparisons $\sim 63$ vs $\sim 57 \%$ correct for the posterior ROI, and $\sim 59$ vs $\sim 53 \%$ correct for the anterior ROI).

\section{Quantification of image changes}

Generalisation of classification performance across image transformations, as shown here, could arise through abstract representation by the neuronal populations in LOC, but could also to some degree reflect overlap in low-level visual features. To better understand the implications of our findings from LOC, we quantified the degree to which our images of objects differed in their physical (low-level) image properties. We calculated a pixelwise dissimilarity measure (Euclidean distance) as performed previously (e.g., Grill-Spector et al., 1999; Vuilleumier, Henson, Driver, \& Dolan, 2002) for all relevant stimulus comparisons. The mean (across pairwise comparisons) and SD pixelwise change was $78.4+$ / -6.6 between category, $70.6+/-9.2$ within category, $78.1+/-11.8$ between same objects in different sizes and $52.7+/-13.1$ between same objects in different views. The pixelwise change induced by different sizes was thus larger than that induced by exemplar change (and even comparable to that induced by different categories), but the difference due to view changes was smaller than that due to different exemplars. The pattern of discrimination performance obtained in LOC for objects, sizes and views is therefore not completely explained by the pixelwise similiarity of the stimuli, since in the size experiment, the pattern of results for discrimination of objects versus sizes is opposite to to one expected on the basis of this similarity measure.

In addition, we simulated generalisation performance by entering the stimulus images themselves into an SVM and testing whether object images could be correctly classified on the basis of their pixelwise physical characteristics alone (see O'Toole, Jiang, Abdi, \& Haxby, 2005, for a similar classification analysis of stimulus images). Across changes in size, object stimuli could be identified in 32 of 48 cases (67\%) and across changes in view in 47 of 48 cases (98\%). The reference value for identical images in these cases is $100 \%$ correct. These findings demonstrate that generalisation in SVM classification can occur based on a low-level pixel-wise representation of the stimuli (in spite of the pixel-wise change on average across all size comparisons being bigger than across all object comparisons, see above). However, these results also indicate that generalisation based on 
such a representation does not reach the same level of discrimination accuracy as for identical images for the size changes used here.

\section{Object discrimination in early visual cortex}

Finally, we also analysed responses of early visual cortex to determine whether the pattern of discrimination performance there was different from the one obtained in LOC. Patterns of activation in early visual cortex discriminate between physically different simple stimuli (e.g., Kamitani \& Tong, 2005; Haynes \& Rees, 2005), so we anticipated that these regions would also discriminate between the different objects used here (since different shape is invariably associated with different local properties, that may be efficiently discriminated by the small receptive fields of early visual cortical regions). However, the extent to which early visual responses would also generalise across the size and view changes in Experiments $2 \& 3$ was less clear, and therefore of more general interest.

We assessed brain responses within a new region of interest defined by a mask derived from a probabilistic map of Brodmann area 17 that therefore identified regions of cortex consistent with primary visual cortex (see Methods for details). Results are summarised in Figure 7 (reported here for identical numbers of voxels as also used in the analysis of LOC, while inclusion of more voxels did not further improve classification accuracies). In Experiment 1, reliable above chance discrimination performance was observed for both comparisons across (e.g. for 400 voxels $\mathrm{t}(5)=8.5, \mathrm{p}<.001$ ) and within (e.g. for 400 voxels $\mathrm{t}(5)=5.0, \mathrm{p}<.01)$ category, accuracies being higher for comparisons across than within category (for 400 voxels $t(5)=8.4, p<.001$ ) In Experiment 2, both discrimination of same images (for 400 voxels $t(5)=8.3, p<.001$ ) and generalisation across size (for 400 voxels $\mathrm{t}(5)=8.4, \mathrm{p}<.001)$ were above chance, however with a marked difference in accuracy level (higher for test on data from identical than different image, at 400 voxels $t(5)=6.5, \mathrm{p}<.01$ ).

Importantly, size discrimination yielded even higher prediction accuracies than object discrimination (see Figure 7, difference between size and object discrimination at 400 voxels $t(5)=4.1, p<.0 .01)$. These results fit well with our quantification of pixelwise stimulus similarity as presented above, and show that the simple measure of image dissimilarity used here may account for much of the discrimination performance obtained in this region. Finally, in Experiment 3, above chance discrimination performance was observed for discrimination of object exemplars in same (for 400 voxels $\mathrm{t}(5) 6.4, \mathrm{p}<.01$ ) and different (for 400 voxels $\mathrm{t}(5) 4.6, \mathrm{p}<.01$ ) views, at a comparable level of accuracy. Discrimination of view, although also above chance (for 400 voxels $t(5) 2.8, p<.05$ ), was lower than object discrimination (difference between view and object discrimination, for 400 voxels $t(5) 4.6, p$ $<.01)$.

These results indicate that not only object discrimination based on identical images, but also object discrimination across changes in size and view (although not always at comparable levels of accuracy as for identical images, see size experiment) was possible based on activity patterns of early visual cortex. However, the pattern of findings in early visual cortex was partially distinct from that in LOC. While early visual responses allowed better discrimination of different sizes of the same object than different objects in the same size, the opposite pattern was found in LOC, as confirmed by a highly significant interaction between region and type of discrimination (object vs size), $F(1,5)=43.1, p<.001$. The fact that the same pattern was not observed for comparison of object versus view discrimination may be related to the greater pixelwise similarity of the different views used here. 


\section{Discussion}

In three independent experiments, we tested whether distributed fMRI signals sampled from the functionally defined human LOC represented information that supported discrimination between individual objects within a category, in addition to discrimination between categories. Using our pattern-based multivariate analysis on fMRI signals, we could successfully use signals sampled from LOC not only to predict the category of a given object, as done previously, but also discriminate between exemplars within the same category. Moreover, successful discrimination of individual category exemplars was possible when different stimulus sizes and viewing angles were used for training and test. Discrimination of objects as well as generalisation across the size and view changes was also possible based on early visual cortex activity with high accuracy, but differences between LOC and early visual regions we found in the detailed pattern of results for discrimination of different properties (notably objects versus size).

Our results provide further support for with the idea that distributed information about object categories is represented in ventral occipitotemporal cortex (Haxby et al., 2001; Carlson, Schrater, \& He, 2003; Cox \& Savoy, 2003, O'Toole, Jiang, Abdi, \& Haxby, 2005). Beyond this, we show that such distributed discriminatory information is not limited to the relatively coarse shape differences between different categories (which are accompanied by additional semantic differences), but is also found within a single category. Our results are obtained with a limited number of categories and exemplars due to the constraints imposed by the current level of sensitivity of fMRI that restricts the number of experimental conditions, and further work will be needed to show in how far these results generalise to further object categories and exemplars. Overall prediction accuracies in our study were somewhat lower than those found in previous studies using the multivariate approach in relation to object categories (Haxby et al., 2001; Carlson, Schrater, \& He, 2003; Cox \& Savoy, 2003, O'Toole, Jiang, Abdi, \& Haxby, 2005). This may be related to the specific categories used, differences in experimental design (short mini-blocks of only 4 objects here), or maybe most importantly, due to our use of an orthogonal task (colour judgement). We chose this task to avoid task-by-condition confounds, but the thereby reduced attention to object shape might have weakened object-related activity. The effects described here are therefore most likely a conservative estimate of discriminability of single-object response patterns.

Our findings are also informative as to why investigations that based their analyses on the mean level of activity within a region of interest (see e.g., Malach et al., 1995; Grill-Spector, 2003) failed to detect selectivity for different types of objects in the same cortical areas. Our analyses indicate that discrimination of categories (and exemplars) is possible when using patterns of activity across multiple voxels, but not for predictions based upon mean activity or the few most selective voxels in the case of exemplar discrimination (Experiment 1). Prediction accuracies increased with the number of voxels included, compatible with the notion that also within the same category, representation of object shape is not restricted to the most discriminative voxels (as proposed in the distributed representation model for object categories, Ishai, Ungerleider, Martin, Schouten, \& Haxby, 1999; Haxby et al., 2001).

A more detailed analysis of our data investigated activity sampled from posterior (lateral occipital) and anterior (fusiform) subregions of the LOC. Across the three experiments, the overall level of prediction accuracy was higher for posterior compared to anterior LOC. In the first experiment, prediction accuracies were also higher for between-category than within-category comparisons, resulting in within-category comparisons being close to chance in the anterior LOC. These data are somewhat inconsistent with the notion of a simple object processing hierarchy according to which more posterior subregions would just be responsive to any object, potentially subserving "object detection", while more anterior 
regions in ventral temporal cortex (as also our anterior ROI) contain subregions discriminative between objects (see Malach et al., 1995; Grill-Spector, 2003). Our results show that the posterior object responsive regions located in lateral/inferior occipital cortex alone can discriminate not only between categories but also exemplars at the level of distributed responses, while some previous work, (see Ishai, Ungerleider, Martin, \& Haxby, 2000) already suggested differential responses to categories like faces, houses, and chairs in similar locations in ventral occipito-temporal cortex.

Whether the anterior-posterior difference observed here reflects a physiological difference in encoding (of processing converging more onto category prototypes than individual exemplars in anterior regions) cannot be determined on the basis of our data alone. We did not observe any interaction between region and stimulus condition in between versus within category performance, however, since discrimination of exemplars was close to chance in the anterior LOC, this could reflect a floor effect. Thus our results need not imply that identity information (and potential invariance to size and view) is not represented in the anterior regions; rather, that it was merely not detectable with the methods used here. Differences could exist in the BOLD signal-to-noise characteristics between the two regions (also see Figure 2 for differences in the overall visual response between the occipital and ventral temporal subparts; and note that the overall difference in level of prediction accuracies between early visual cortex and LOC is likely to result from differences in signalto-noise). Other contributing factors to any differences in classification performance may include adaptation across repetitions of the same stimuli (potentially more pronounced in the anterior region), or differences in the spatial scale of the underlying object selectivities. Any variation in this spatial layout would be sampled in a more or less efficient way at a particular MRI resolution, leading to differences in performance of the multivariate classification algorithm (Haynes \& Rees, 2005). Future studies using higher scanning resolution and/or different paradigms may disambiguate between these potential explanations. Little is currently known about the spatial scale of object selectivies in human cortex, while monkey neurophysiology suggests that a column-like selectivity for object features might exist in the order of $500 \mu \mathrm{m}$ (Fujita, Tanaka, Ito, \& Cheng, 1992). In this case (assuming no limitations in the spatial resolution of the BOLD effect itself) higher spatial resolution should improve classification performance, but even $1 \mathrm{~mm}$ sampling resolution would still not resolve the true underlying pattern of object selectivities, but rely on partial volume effects.

The finding that early visual cortex could also discriminate between the different object images used here was expected and is in line with previous studies using object categories (Cox \& Savoy, 2003) who reported the highest classification accuracies for discrimination of categories when the classifier was allowed to select voxels from multiple areas including low-level visual cortex, as opposed to an analysis on high-level object responsive areas only. Finding above chance discrimination of activity patterns for objects in a given brain region thus need not necessarily reflect processing at the level of objects (instead of low-level local properties of the images). Beyond using pattern recognition to only quantify separability of responses to different conditions in this way, our experiments constitute a first step into the direction of using these techniques for the more challenging task of understanding mechanisms of representation of visual information at different stages of the processing pathway. The fact that we observed above-chance generalisation of classification across sizes and views in LOC, but also, less expected, in early visual cortex, suggests that generalisation of the classifier, although necessary, is not alone sufficient to conclude invariant representation by the neuronal population. Generalisation further need not imply that the patterns for both conditions are identical, but may arise based on just a few voxels that show overlapping activation, for example in foveal areas. Important additional information is therefore derived from the joint consideration of generalisation across, and 
the discrimination of a given feature. This revealed a dissociation between LOC and early visual cortex in the size experiment, where the relative pattern of accuracies (for discrimination of objects in same or different size, and discrimination of size for the same object) was less well predicted by our quantification of pixel-wise stimulus similarity in LOC than in early visual cortex. Our data therefore support a size-invariant coding of object shape in LOC, but do not allow us to determine whether the representation is any more abstract than this, since given the pixel-wise similarity results in the view experiment, an interpretation of the LOC results in terms of low-level overlap cannot be excluded.

The finding regarding size invariance in LOC may appear to contrast with reports that only a small percentage of single neurons in monkey IT cortex generalise across size or viewpoint (Lueschow, Miller, \& Desimone, 1994; Ito, Tawamura, Fujita, \& Tanaka, 1995; Logothetis, Pauls, \& Poggio, 1995; Booth \& Rolls, 1998). However, these studies measured invariance of stimulus selectivity at the single-neuron level, which does not exclude invariance expressed at the population level, that may be measurable with fMRI. Although in the data shown here, the different sizes of objects (in spite of their higher pixelwise dissimilarity) were not discriminated in LOC, the possibility remains that higher spatial sampling (or using even larger changes in stimulus size) would reveal independent information about object shape and size also in LOC. Indeed, such independent coding is suggested by recent findings that employed pattern recognition methods on populations of single neurons in monkey IT cortex (Hung, Kreiman, Poggio, \& DiCarlo, 2006), and this would also be compatible with fMRI results that show some retinotopic organisation in area LO, albeit weaker than in earlier areas (Larsson \& Heeger, 2006).

Previous imaging studies investigating size and/or viewpoint invariance of object representation using paradigms based on adaptation/priming provided findings that range from size-invariance being more pronounced in (or restricted to) anterior parts of LOC (Grill-Spector et al., 1999; Sawamura, Georgieva, Vogels, Vanduffel, \& Orban, 2005), through size-invariance in occipito-temporal cortex and view-invariance only in more anterior regions of the left fusiform gyrus (Vuilleumier, Henson, Driver, \& Dolan, 2002) to view-invariance in bilateral fusiform (but not posterior LO) regions (James, Humphreys, Gati, Menon, \& Goodale, 2002). Here, we relied on aspects of BOLD activity that were constant across multiple replications of the same stimulus condition, rather than repetitionassociated changes. Our results provide converging evidence for size invariance in LOC, however, using this alternative approach, we found evidence for generalisation across size, and inferior discrimination for sizes as compared to objects, already in the posterior subpart of LOC. One possibility is that an overall weaker adaptability in the posterior as compared to anterior LOC (Sawamura, Georgieva, Vogels, Vanduffel, \& Orban, 2005) might account for the fact that some previous studies using repetition paradigms did not detect generalisation of adaptation across size changes in that region.

Beyond differences associated with paradigms, it is likely that object discrimination and generalisation of neuronal responses across stimulus attributes as size and view is not absolute, but depends on stimulus change in a gradual manner not only in earlier, but also in higher areas, as also indicated by behavioural research (Lawson, 1999; Tarr \& Bulthoff, 1998; Ashbridge \& Perrett, 1998). We employed moderate changes in our first experiments presented here, which are, however, within the range used in previous studies (Grill-Spector et al., 1999; Vuilleumier, Henson, Driver, \& Dolan, 2002; James, Humphreys, Gati, Menon, \& Goodale, 2002), to ensure that the chosen manipulation did not result in, for example, strongly unusual views, or occlusion of parts. More studies will be required to reveal the extent of generalisation ("tuning") of fMRI response patterns across a wider range of changes, and across a broader range of (potentially parametrised) changes in object shape. Increasing the spatial resolution of the MRI acquisition, together with further development 
of methodological aspects, should provide additional sensitivity to investigate more subtle aspects of shape representation across a higher number of experimental conditions.

Therefore, the pattern recognition approach as used here could in the future prove fruitful to approach the most interesting question of the nature of structural primitives (e.g., image-like "views", or more structured part-based elements, see Biederman \& Kalocsai, 1997; Bulthoff, Edelman, \& Tarr, 1995; Riesenhuber \& Poggio, 1999) underlying the representation of the visual input at different levels of the cortical hierarchy.

\section{Supplementary Material}

Refer to Web version on PubMed Central for supplementary material.

\section{Acknowledgments}

This research was supported by the Wellcome Trust.

We thank Richard Henson and Joy Geng for comments on the manuscript.

\section{References}

Ashbridge, E.; Perrett, DI. Generalising across object orientation and size. In: Walsh, V.; Kulikowski, J., editors. Perceptual constancy. Why things look as they do. Cambridge University Press; Cambridge: 1998. p. 192-209.

Avidan G, Hasson U, Hendler T, Zohary E, Malach R. Analysis of the neuronal selectivity underlying low fMRI signals. Current Biology. 2002; 12:964-972. [PubMed: 12123569]

Biederman I, Kalocsai P. Neurocomputational bases of object and face recognition. Philosophical Transactions of the Royal Society of London B. 1997; 352:1203-19.

Booth MCA, Rolls ET. View-invariant representation of familiar objects by neurons in the inferior temporal visual cortex. Cerebral Cortex. 1998; 8:510-523. [PubMed: 9758214]

Bulthoff HH, Edelman SY, Tarr MJ. How are three-dimensional objects represented in the brain? Cerebral Cortex. 1995; 5:247-60. [PubMed: 7613080]

Carlson TA, Schrater P, He S. Patterns of activity in the categorical representation of objects. Journal of Cognitive Neuroscience. 2003; 15:704-717. [PubMed: 12965044]

Christianini, N.; Shawe-Taylor, J. An introduction to support vector machines and other kernel-based learning methods. Cambridge University Press; Cambridge: 2000.

Cox D, Savoy R. Functional magnetic resonance imaging (fMRI) "brain reading": Detecting and classifying distributed patterns of fMRI activity in human visual cortex. Neuroimage. 2003; 19:261270. [PubMed: 12814577]

Eger E, Henson RN, Driver J, Dolan RJ. BOLD repetition decreases in object responsive ventral visual areas depend on spatial attention. Journal of Neurophysiology. 2004; 92:1241-1247. [PubMed: 15056686]

Fujita I, Tanaka K, Ito M, Cheng K. Columns for visual features in monkey inferotemporal cortex. Nature. 1992; 26:301-302.

Grill-Spector K. The neural basis of object perception. Current Opinion in Neurobiology. 2003; 13:159-166. [PubMed: 12744968]

Grill-Spector K, Henson R, Martin A. Repetition and the brain: neural models of stimulus-specific effects. Trends in Cognitive Sciences. 2006; 10:14-23. [PubMed: 16321563]

Grill-Spector K, Kushnir T, Edelman S, Avidan G, Itzchak Y, Malach R. Differential processing of objects under various viewing conditions in the human lateral occipital complex. Neuron. 1999; 24:187-203. [PubMed: 10677037]

Gross CG. Representation of visual stimuli in inferior temporal cortex. Philosophical Transactions of the Royal Society of London B. 1992; 335:3-10. 
Haxby JV, Gobbini MI, Furey ML, Ishai A, Schouten JL, Pietrini P. Distributed and overlapping representation of faces and objects in ventral temporal cortex. Science. 2001; 293:2425-2430. [PubMed: 11577229]

Haynes J-D, Rees G. Predicting the orientation of invisible stimuli from activity in human primary visual cortex. Nature Neuroscience. 2005; 8:686-691.

Henson RN. Neuroimaging studies of priming. Progress in Neurobiology. 2003; 70:53-81. [PubMed: 12927334]

Henson RN, Shallice T, Gorno-Tempini ML, Dolan RJ. Face repetition effects in implicit and explicit memory tests as measured by fMRI. Cerebral Cortex. 2002; 12:178-186. [PubMed: 11739265]

Hung CP, Kreiman G, Poggio T, DiCarlo JJ. Fast readout of object identity from macaque inferior temporal cortex. Science. 2005; 310:863-866. [PubMed: 16272124]

Ishai A, Ungerleider LG, Martin A, Haxby JV. The representation of objects in the human occipital and temporal cortex. Journal of Cognitive Neuroscience. 2000; 12:35-51. [PubMed: 11506646]

Ishai A, Ungerleider LG, Martin A, Schouten JL, Haxby JV. Distributed representation of objects in the human ventral visual pathway. Proceedings of the National Academy of Sciences USA. 1999; 96:9379-9384.

Ito M, Tawamura H, Fujita I, Tanaka K. Size and position invariance of neuronal responses in monkey inferotemporal cortex. Journal of Neurophysiology. 1995; 73:218-226. [PubMed: 7714567]

James TW, Humphrey GK, Gati JS, Menon RS, Goodale MA. Differential effects of viewpoint on object-driven activation in dorsal and ventral streams. Neuron. 2002; 35:793-801. [PubMed: 12194877]

Kamitani Y, Tong F. Decoding the visual and subjective contents of the human brain. Nature Neuroscience. 2005; 8:670-785.

Kourtzi Z, Kanwisher N. Cortical regions involved in perceiving object shape. Journal of Neuroscience. 2000; 20:3310-3318. [PubMed: 10777794]

Kourtzi Z, Kanwisher N. Representation of perceived object shape by the human lateral occipital complex. Science. 2001; 293:1506-1509. [PubMed: 11520991]

Krekelberg B, Boynton GM, van Wezel RJA. Adaptation: from single cells to BOLD signals. Trends in Neurosciences. 2006; 29:251-256.

Larsson J, Heeger DJ. Two retinotopic visual areas in human lateral occipital cortex. Journal of Neuroscience. 2006; 26:13128-13142. [PubMed: 17182764]

Lawson R. Achieving visual object constancy across plane rotation and depth rotation. Acta Psychologica. 1999; 102:221-245. [PubMed: 10504882]

Logothetis NK, Pauls J, Poggio T. Shape representation in the inferior temporal cortex of monkeys. Current Biology. 1995; 5:552-563. [PubMed: 7583105]

Lueschow A, Miller EK, Desimone R. Inferior temporal mechanisms for invariant object recognition. Cerebral Cortex. 1994; 4:523-531. [PubMed: 7833653]

Malach R, Reppas JB, Benson RR, Kwong KK, Jiang H, Kennedy WA, Ledden PJ, Brady TJ, Rosen $\mathrm{BR}$, Tootell RB. Object-related activity revealed by functional magnetic resonance imaging in human occipital cortex. Proceedings of the National Academy of Science USA. 1995; 92:81358139.

Murray SO, Wojciulik E. Attention increases neural selectivity in the human lateral occipital complex. Nature Neuroscience. 2004; 7:70-74.

O'Toole A, Jiang F, Abdi H, Haxby JV. Partially distributed representation of objects and faces in ventral temporal cortex. Journal of Cognitive Neuroscience. 2005; 17:580-590. [PubMed: 15829079]

Riesenhuber M, Poggio T. Hierarchical models of object recognition in cortex. Nature Neuroscience. 1999; 2:1019-1025.

Rolls ET, Tovee MJ. Sparseness of the neuronal representation of stimuli in the primate temporal visual cortex. Journal of Neurophysiology. 1995; 73:713-726. [PubMed: 7760130]

Sawamura H, Georgieva S, Vogels R, Vanduffel W, Orban GA. Using functional magnetic resonance imaging to assess adaptation and size invariance of shape processing by humans and monkeys. Journal of Neuroscience. 2005; 25:4294-4306. [PubMed: 15858056] 
Tarr, MJ.; Bulthoff, HH. Image-based recognition in man, monkey, and machine. In: Tarr, MJ.; Bulthoff, HH., editors. Object recognition in man, monkey, and machine. MIT Press; Cambridge: 1998. p. $1-20$.

Vapnik, V. The nature of statistical learning theory. Springer Verlag; Berlin: 1995.

Vuilleumier P, Henson RN, Driver J, Dolan RJ. Multiple levels of visual object constancy revealed by event-related fMRI of repetition priming. Nature Neuroscience. 2002; 5:491-495.

Vuilleumier P, Schwartz S, Duhoux S, Dolan RJ, Driver J. Selective Attention Modulates Neural Substrates of Repetition Priming and "Implicit" Visual Memory: Suppressions and Enhancements Revealed by fMRI. Journal of Cognitive Neuroscience. 2005; 17:1245-1260. [PubMed: 16197681]

Yi D-J, Chun MM. Attentional Modulation of Learning-Related Repetition Attenuation Effects in Human Parahippocampal Cortex. Journal of Neuroscience. 2005; 25:3593-3600. [PubMed: 15814790]

Young MP, Yamane S. Sparse population coding of faces in the inferotemporal cortex. Science. 1992; 256:1327-1331. [PubMed: 1598577] 

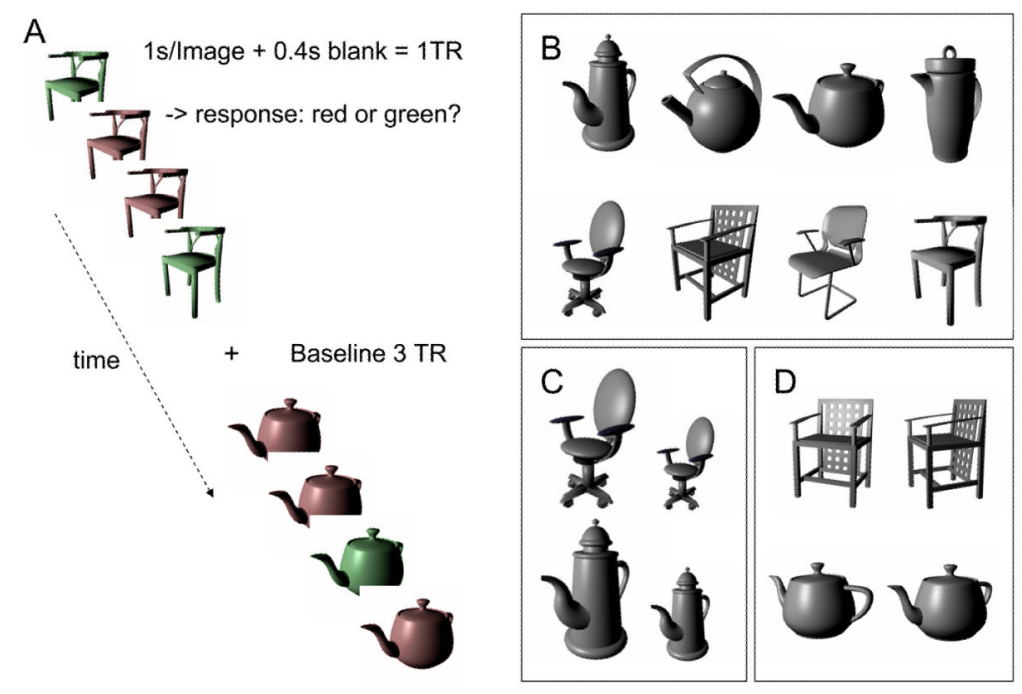

Figure 1.

Stimuli and experimental design. Across all three experiments, stimuli were presented in short blocks with four successive presentations of the same object, separated by short baseline periods (A). Participants were required to respond to each picture depending on the colour of the object, which could randomly appear in a red or green hue. Two everyday man-made categories (teapots and chairs) with 4 different exemplars each served as stimuli (B) These were created from 3D-models with a unified surface texture and lighting. In Experiment 2, objects were presented in two different sizes (C), and in two different views (D) in Experiment 3. 

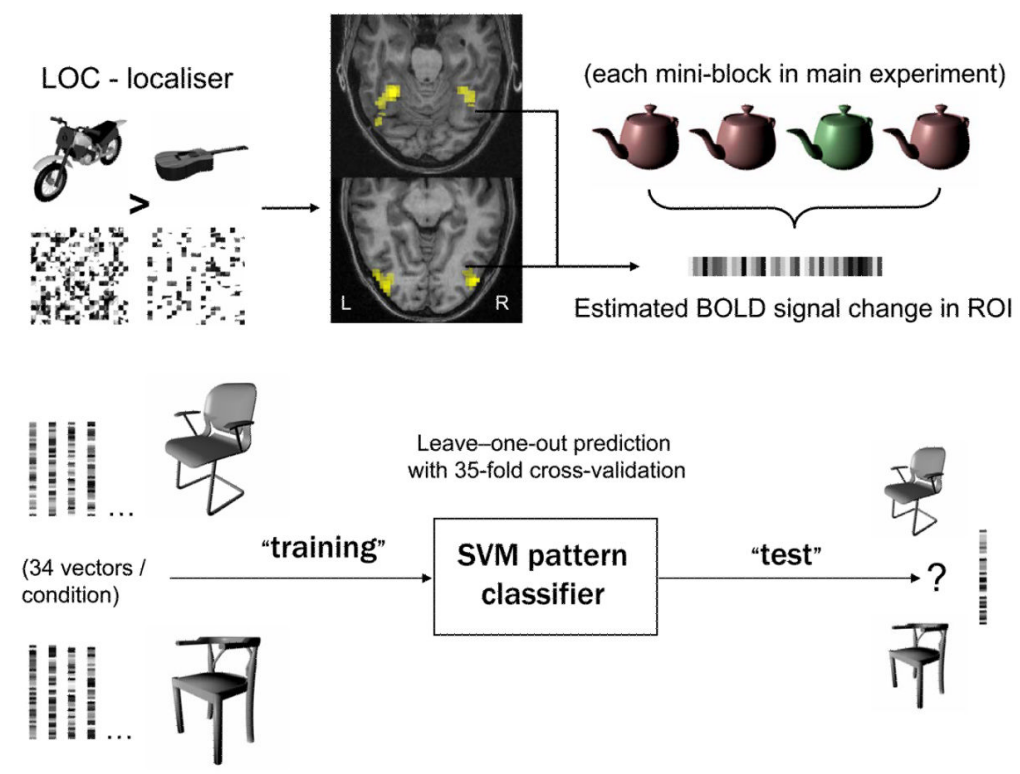

Figure 2.

Schematic illustration of analysis procedure (A). Based on a standard LOC localiser comparing pictures of different everyday objects to scrambled versions of the same stimuli, regions of interest were defined in each participant, corresponding to the left and right posterior (lateral/inferior occipital) and anterior (ventral temporal) subparts of the lateral occipital complex. Estimates of BOLD signal change were obtained for this ROI in the 3 main experiments, corresponding to regression coefficients for each of the short blocks with one given object, convolved with a standard hemodynamic response function. 35 of such independent estimates were obtained for each experimental condition. These 35 multi-voxel pattern vectors for each condition were entered into a classification procedure using SVM, corresponding to pairwise "leave-one-out" prediction with cross-validation (see Methods). B - Group analysis $(\mathrm{n}=18)$ of the LOC-localiser (objects minus scrambled objects) thresholded at $\mathrm{p}<.05$, corrected, for the whole brain, superimposed onto a standard brain in MNI space, displaying group activations for posterior (lateral occipital cortex) and anterior (fusiform/ventral temporal cortex) object responsive areas. MNI coordinates were $48-84-3$ and $-45-840$ for the posterior, and $39-45-24$ and $-24-39-21$ for the anterior subregion. $\mathrm{C}$ - Average responses to objects and scrambled objects (parameter estimates) derived from group maxima (upper panel) and individual maxima (lower panel), indicating stronger responses to objects than scrambled objects in both subregions, while the overall visual response is stronger in the posterior than the anterior subregion. 


\section{Experiment 1:}

discrimination between and within category (across subjects)

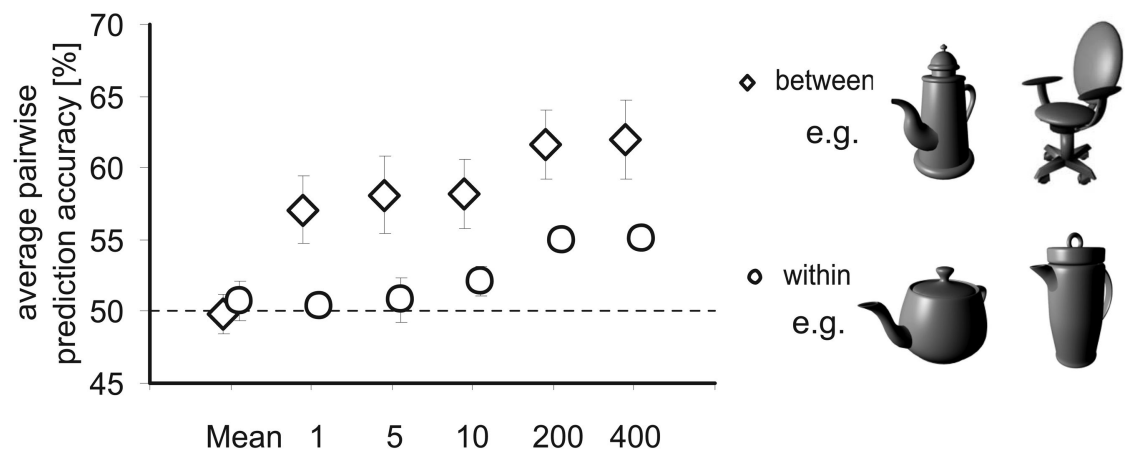

Number of voxels

Figure 3.

Discrimination performance for Experiment 1 (object discrimination between and within category discrimination), displaying means and SEM across 6 subjects for pairwise prediction accuracies (averaged across all possible pairwise between-category and withincategory comparisons, also shown is the simulated "chance" performance obtained with randomised labels of conditions). Accuracies are plotted for prediction based on mean activity across the ROI, and for 1, 5, 10, 200, or 400 voxels, for which voxels were rankordered depending on their t-value for a given pairwise comparison in the training data. Additional results for intermediate voxel numbers can be found in Supplemental Materials. 


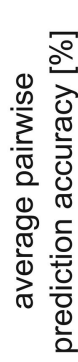

e.g. Training:

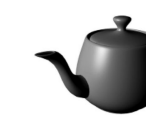

Test:

Same size
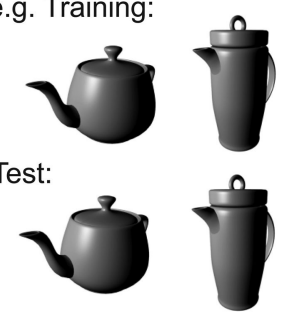

- different size
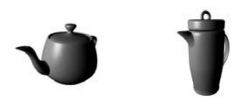

Figure 4.

Discrimination performance for Experiment 2 (object discrimination within category, generalisation across size, and size discrimination), displaying means and SEM across 6 subjects for pairwise prediction accuracies (averaged across all possible pairwise comparisons, also shown is the simulated "chance" performance obtained with randomised labels of conditions). Accuracies are plotted for prediction based on mean activity across the ROI, and for $1,5,10,200$, or 400 voxels, for which voxels were rank-ordered depending on their t-value for a given pairwise comparison in the training data. Additional results for intermediate voxel numbers can be found in Supplemental Materials. 


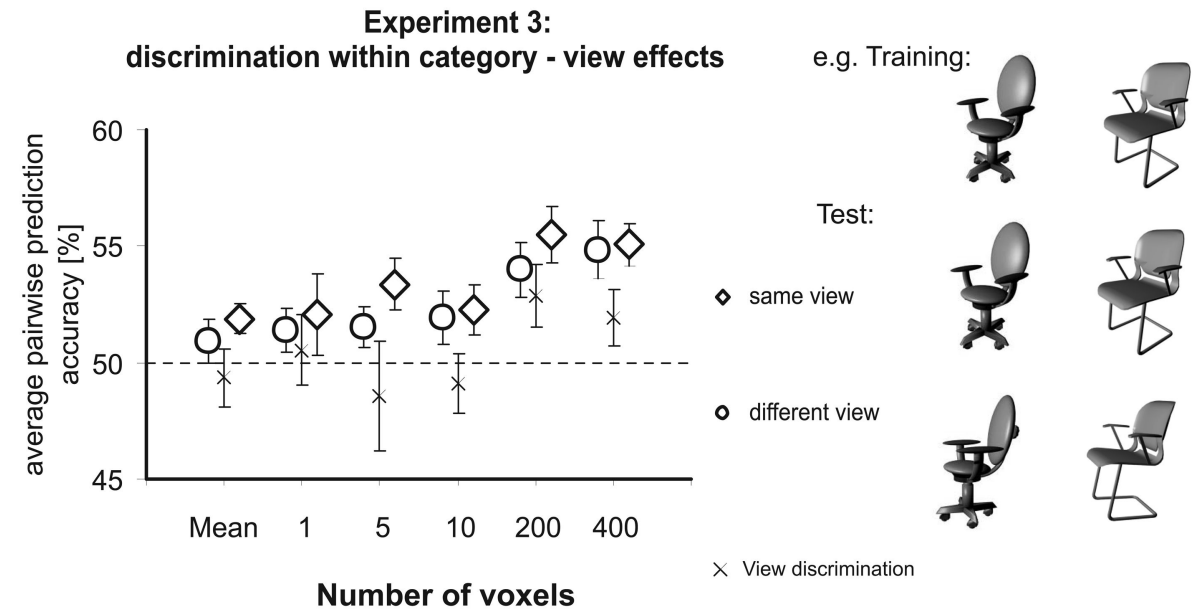

Figure 5.

Discrimination performance for Experiment 3 (object discrimination within category, generalisation across views, and view discrimination), displaying means and SEM across six subjects for pairwise prediction accuracies (averaged across all possible pairwise comparisons, also shown is the simulated "chance" performance obtained with randomised labels of conditions). Accuracies are plotted for prediction based on mean activity across the ROI, and for $1,5,10,200$, or 400 voxels, for which voxels were rank-ordered depending on their t-value for a given pairwise comparison in the training data. Additional results for intermediate voxel numbers can be found in Supplemental Materials. 
A Prediction dependent on ROI - Exp 1

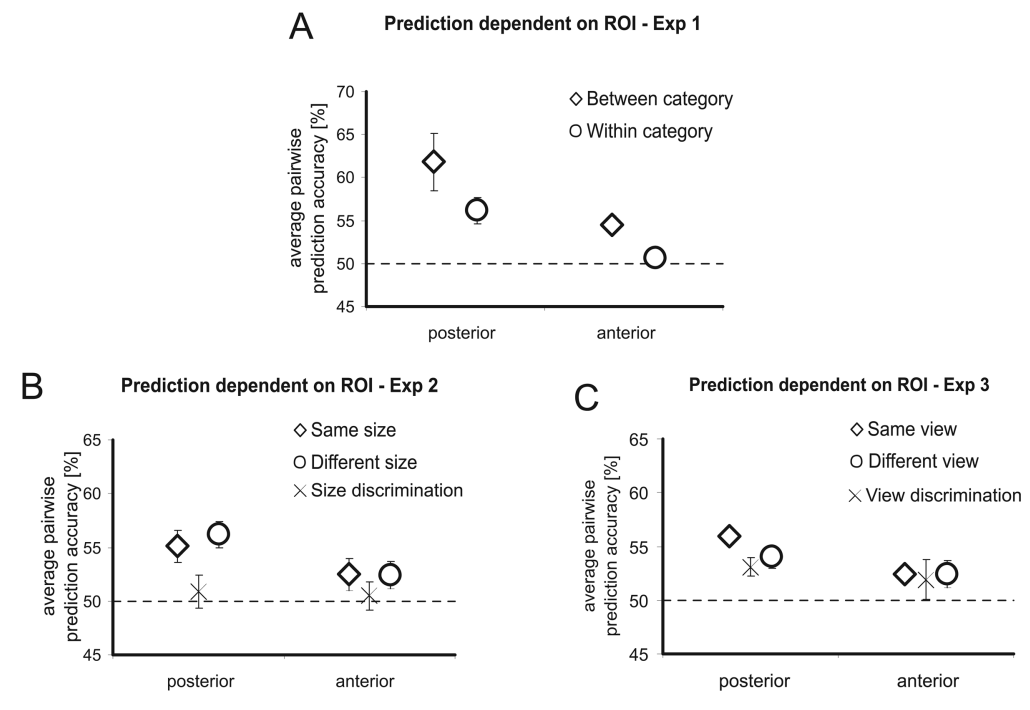

Figure 6.

Prediction accuracies when separately testing posterior (lateral occipital) and anterior (fusiform) parts of the LOC. Consistently better discrimination (across the three experiments A-C) was possible based on MR signal in posterior compared to anterior parts (see text for details and statistics). In these analyses all voxels were included without pre-selection. Means and SEM across 6 subjects are shown in all graphs. 
A

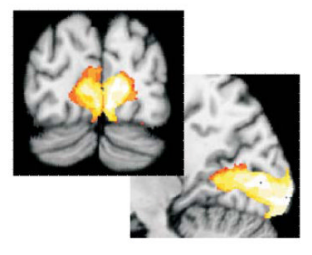

discrimination within category - size effects

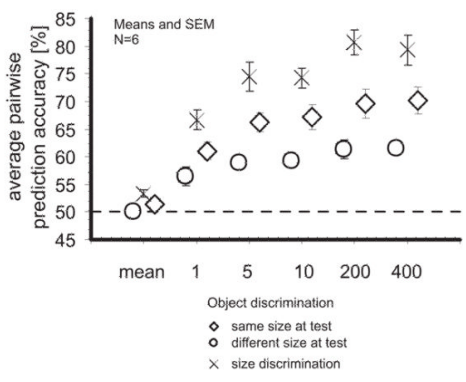

B discrimination between and within category

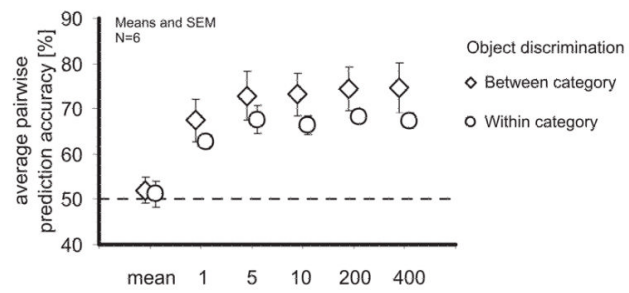

D discrimination within category - view effects

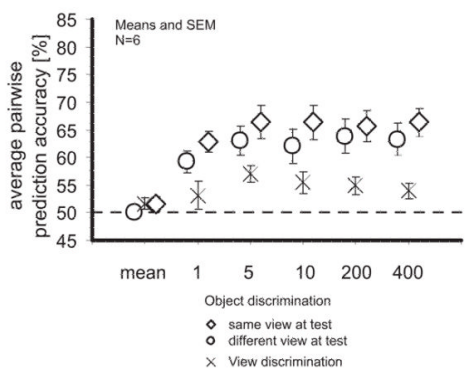

Figure 7.

Prediction accuracies for early visual cortex. A) Region of interest in MNI space (derived from a probabilistic map of area 17/V1 using the anatomy toolbox for SPM) that was transformed back onto individual subjects brains. B) Prediction accuracies for object discrimination across and within category (Experiment 1), means across 6 subjects and SEM. C) Prediction accuracies for object discrimination within category, generalisation across size, and size discrimination (Experiment 2), means across 6 subjects and SEM. D) Prediction accuracies for object discrimination within category, generalisation across size, and size discrimination (Experiment 2), means across 6 subjects and SEM. In all cases, results are plotted for mean activity and the same numbers of voxels (rank-ordered based on pairwise t-test in training data) as used for LOC. Also shown is the simulated "chance" performance obtained with randomised labels of the conditions. Inclusion of further voxels did not increase prediction accuracies for early visual areas. 